\title{
Generic Construction of Consensus Algorithms for Benign and Byzantine Faults (Full Version)
}

\author{
Olivier Rütti Zarko Milosevic André Schiper \\ Ecole Polytechnique Fédérale de Lausanne (EPFL) \\ 1015 Lausanne, Switzerland \\ \{olivier.rutti,zarko.milosevic,andre.schiper\}@epfl.ch
}

\begin{abstract}
The paper proposes a generic consensus algorithm that highlights the basic and common features of known consensus algorithms. The parameters of the generic algorithm encapsulate the core differences between various consensus algorithms, including leader-based and leader-free algorithms, addressing benign faults, authenticated Byzantine faults and Byzantine faults. This leads to the identification of three classes of consensus algorithms. With the proposed classification, Paxos and PBFT indeed belong to the same class, while FaB Paxos belongs to a different class. Interestingly, the classification allowed us to identify a new Byzantine consensus algorithm that requires $n>4 b$, where $b$ is the maximum number of Byzantine processes.
\end{abstract}

\section{Introduction}

Consensus is a fundamental and difficult problem in fault tolerant distributed computing. This explains the numerous consensus algorithms that have been published, with different features and for different fault models. Considering these numerous algorithms, it would be helpful to classify them, in order to identify the basic mechanisms on which they rely. This would allow a better understanding of consensus algorithms, particularly for a classification encompassing benign faults and malicious (Byzantine) faults.

The paper provides such a classification by proposing a generic consensus algorithm, which highlights the basic and common features of known consensus algorithms. The parameters of the generic algorithm encapsulate the core differences between various consensus algorithms, including leader-based and leader-free algorithms, addressing benign faults, authenticated Byzantine faults and Byzantine faults. Instantiations of the parameters allow us to obtain these various algorithms. The generic algorithm also allows us to discuss randomized consensus algorithms.
The generic algorithm consists of successive phases, where each phase is composed of three rounds: a selection round, a validation round and a decision round. The validation round may be skipped by some algorithms, which introduces a first dichotomy among consensus algorithms: those that require the validation round, and the others for which the validation round is not necessary. We further subdivide the former class in two, based on the state variables required. This lead us to identify three classes of consensus algorithms, and tradeoffs between these classes. With this classification, Paxos [11] (benign faults) and PBFT [4] (Byzantine faults) indeed belong to the same class, while $\mathrm{FaB}$ Paxos [16] belongs to a different class. Interestingly, the classification allowed us to identify a new Byzantine consensus algorithm that requires $n>4 b$ (inbetween the requirement $n>5 b$ of FaB Paxos and $n>3 b$ of PBFT). ${ }^{1}$

Our generic algorithm is based on four parameters: the $F L V$ function, the Selector function, the threshold parameter $T_{D}$, and the flag $F L A G(*$ or $\phi)$. The functions $F L V$ and Selector are characterized by abstract properties; $T_{D}$ is defined with respect to $n$ (number of processes), $f$ (maximum number of benign faults) and $b$ (maximum number of Byzantine processes). We can prove correctness of the generic consensus algorithm by referring only to the abstract properties of our parameters. The correctness proof of any specific instantiated consensus algorithm consists simply in proving that the instantiations satisfy the abstract properties of the corresponding functions.

The paper is not the first one to propose a generic consensus algorithm, but it goes beyond previous approaches. Mostéfaoui et al. [18] propose a consensus framework restricted to benign faults, which allows unification of leader oracle, random oracle and failure detector oracle. Guerraoui and Raynal [9] propose a generic consensus algorithm, where generality is encapsulated in a function called $L a m b d a$. The Lambda function encapsulates both our selection and our validation rounds. This does not allow the

\footnotetext{
${ }^{1} b$ is the maximum number of Byzantine processes.
} 
authors of [9] to identify the differences between two of our three classes of consensus algorithms. Moreover, as for [18], the paper is restricted to benign faults. Later, Guerrraoui and Raynal [10] propose a generic version of Paxos in which communication (using shared memory, storage area networks, message passing channels or active disks) is encapsulated in the Omega abstraction. The paper is also restricted to benign faults. Apart from this work, several other authors proposed abstractions related to Paxos-like protocols, e.g., [13] and [14]. Recently, Song et al. [20] proposed building blocks that allow the construction of consensus algorithms. They consider both benign and Byzantine faults. However, they ignore some seminal consensus algorithms such as PBFT and FaB Paxos, and their framework therefore has a somehow limited scope.

The rest of the paper is organized as follows. Section 2 is devoted to the system model and to definitions. Section 3 introduces the generic consensus algorithm and its parameters. Section 4 presents three classes of instantiations for these parameters, and classifies consensus algorithms such as Paxos, FaB Paxos, PBFT into these classes. Section 5 gives examples of instantiations of the generic algorithm. In Section 6 we show how the generic algorithm can be adapted to include randomized consensus algorithms, and Section 7 concludes the paper.

\section{Model and Definitions}

\subsection{System Model}

We consider a variant of a partially synchronous system [7]: we assume that the system alternates between good periods (during which the system is synchronous) and bad periods (during which the system is asynchronous). We differentiate honest processes that execute algorithms faithfully, from Byzantine processes [12], that exhibit arbitrary behavior. Honest processes can be correct or faulty. An honest process is faulty if it eventually crashes, and is correct otherwise. Among the $n$ processes in our system, we assume at most $b$ Byzantine processes and at most $f$ faulty (honest) processes. The set of all processes is denoted by $\Pi$, the set of honest processes by $\mathcal{H}$ and the set of correct processes by $\mathcal{C}$.

Round Model. Distributed algorithms can be expressed as a sequence of rounds. In each round $r$, a process $p$ sends a message to a subset of processes according to a "sending" function $S_{p}^{r}$, and at the end of this round, computes a new state according to a "transition" function $T_{p}^{r}$ that takes as input the vector of messages it received at round $r$ and its current state. Note that this implies that a message sent in round $r$ can only be received in round $r$ (rounds are $c l o s e d$ ).

Honest processes cannot be impersonated: if an honest process receives $v$ from $p$ in round $r$, and $p$ is honest, then $p$ sent $v$ in round $r$. The state of process $p$ in round $r$ is denoted by $s_{p}^{r}$; the message sent by an honest ${ }^{2}$ process is denoted by $S_{p}^{r}\left(s_{p}^{r}\right)$; messages received by process $p$ in round $r$ are denoted by $\vec{\mu}_{p}^{r}$ ( $\vec{\mu}_{p}^{r}[q]$ is the message received from $q$ ).

Communication Predicates. During good periods of our partially synchronous system, we assume the following two communication predicates that are sufficient to solve consensus: $\mathcal{P}_{\text {good }}$ and $\mathcal{P}_{\text {cons }}$. The predicate $\mathcal{P}_{\text {good }}$ ensures that correct processes receive every message sent by a correct process:

$$
\mathcal{P}_{\text {good }}(r) \equiv \forall p, q \in \mathcal{C}: \vec{\mu}_{p}^{r}[q]=S_{q}^{r}\left(s_{q}^{r}\right)
$$

An implementation of $\mathcal{P}_{\text {good }}$ on top of the basic partially synchronous system model with benign and Byzantine faults has been proposed in [7].

The predicate $\mathcal{P}_{\text {cons }}$ provides the same guarantees as the predicate $\mathcal{P}_{\text {good }}$, but additionally ensures that each correct process receives the same set of messages. In the benign fault model (i.e., $b=0$ ), this predicate can be implemented using the implementation of $\mathcal{P}_{\text {good }}$ described in [7] if we assume that no crash occurs in good periods. In the Byzantine fault model (i.e., $b \neq 0$ ), several implementations of $\mathcal{P}_{\text {cons }}$ have been proposed [17, 2]:

$$
\mathcal{P}_{\text {cons }}(r) \equiv \mathcal{P}_{\text {good }}(r) \wedge \forall p, q \in \mathcal{C}: \vec{\mu}_{p}^{r}=\vec{\mu}_{q}^{r}
$$

Based on these definitions, we define the notion of a good phase. A phase is a sequence of rounds. A good phase $\phi$ of $k$ rounds is defined as a phase such that $\mathcal{P}_{\text {cons }}$ holds in the first round, and $\mathcal{P}_{\text {good }}$ holds in the remaining $k-1$ rounds.

\subsection{Unifying Byzantine Faults}

Two different models for Byzantine faults have been considered in literature [7]: (1) authenticated Byzantine faults, where messages can be signed by the sending process (with the assumption that signatures cannot be forged by any other process), and (2) Byzantine faults, where there is no mechanism for signatures (but the receiver of a message knows the identity of the sender). ${ }^{3}$

As shown in [17], the predicate $\mathcal{P}_{\text {cons }}$ allows the unification of these two fault models: (i) $\mathcal{P}_{\text {cons }}$ allows us to express a generic consensus algorithm that is the same for both fault models, and (ii) $\mathcal{P}_{\text {cons }}$ can be implemented out of $\mathcal{P}_{\text {good }}$ in the two fault models. The implementation in the authenticated Byzantine fault model is simpler and requires two rounds; three rounds are needed in the Byzantine fault

\footnotetext{
${ }^{2}$ Note that referring to the state of a Byzantine process does not make sense.

${ }^{3}$ In [12], these models are respectively called (1) Byzantine faults with signed messages, and (2) Byzantine faults with oral messages.
} 
model. To summarize, the predicate $\mathcal{P}_{\text {cons }}$ allows us to describe consensus algorithms without making difference between authenticated Byzantine faults and Byzantine faults. Therefore, in the paper we use the term Byzantine faults for both fault models, except if explicitly mentioned.

\subsection{The Consensus Problem}

In the consensus problem, each process starts with a given initial value, and later possibly decides on a value. The problem is specified by the following properties:

- Agreement: No two honest processes decide differently;

- Termination: All correct processes eventually decide;

- Validity: If all processes are honest and if an honest process decides $v$, then $v$ is the initial value of some process;

- Unanimity [20]: If all honest processes have the same initial value $v$ and an honest process decides, then it decides $v$.

Unanimity (which extends validity) is optional, and only makes sense with Byzantine processes.

Locked Value. In the context of a consensus algorithm, we refer below to the notion of locked value. ${ }^{4}$ This notion has similarities with the notion of univalent configuration defined in [8], but is actually different (see below). A value $v$ is locked in round $r$ if:

1. An honest process has decided $v$ in round $r^{\prime}<r$, or

2. All honest processes have the same initial value $v$.

Item 2 is meaningful only if unanimity must be ensured, or if all processes are honest. In all other cases, item 2 can be ignored. From this definition it follows that, if $v$ is locked in the context of a consensus algorithm then the configuration is $v$-valent. However, the opposite is not true (e.g., if a configuration is $v$-valent in round $r$, and the first honest process $p$ decides $v$ in round $r^{\prime} \geq r$, then $v$ is not locked in round $r$, but only in round $r^{\prime}+1>r$ ).

\section{A Generic Consensus Algorithm}

We now present a generic algorithm (see Algorithm 1), from which well-known consensus algorithms can be instantiated (see Section 5). Generality is obtained by parametrization of Algorithm 1: parameters appear in a box.

\subsection{Generic Algorithm}

The generic Algorithm 1 consists of a sequence of phases that can be seen as successive trials to decide on a value. Each phase $\phi$ consists of three rounds, respectively called selection round $(r=3 \phi-2)$, validation round

\footnotetext{
${ }^{4}$ Note that the definition of the term locked value in some other works differs from our definition.
}

$(r=3 \phi-1)$, and decision round $(r=3 \phi)$. We will see that some values of the parameters allow us to skip the validation round. We first describe process states, and then the three rounds.

Process State. The state of each process $p$ is defined by three variables. Some instantiations of the generic Algorithm 1 do not need all three variables.

Variable vote $_{p}$ represents the value considered for decision by process $p$. This variable is initialized with the initial value $i n i t_{p}$ of process $p$. Variable $t s_{p}$ represents the most recent phase in which the vote of process $p$ has been validated during the validation round. Variable histor $y_{p}$ is a list of pairs $(v, \phi)$, each pair denoting that vote $_{p}$ has been set to $v$ in the selection round of phase $\phi$. In the context of Byzantine processes, variable history $y_{p}$ is used to prove that some value $v$ may have been validated in some phase $\phi$; in the context of benign faults, the variable history $y_{p}$ can be ignored. The size of variable history $y_{p}$ is unbounded, ${ }^{5}$ and both $t s_{p}$ and histor $y_{p}$ can be ignored in some instantiations of our generic algorithm.

The Selection Round ( $r=3 \phi-2)$. The selection round has two roles. First, it allows processes to elect a set of processes, called validators, that have a special role in the next validation round. The election is based on the proposal of each process, which is locally returned by the function Selector $(p, \phi)$. The function Selector $(p, \phi)$ outputs a set of processes $S \subseteq \Pi$ and is formally defined in Section 3.2.

The second role of the selection round is for validators to select a value that will be considered for the decision. The selection is implemented by the function $F L V\left(\vec{\mu}_{p}^{r}\right)$ (stands for "Find the Locked $V$ alue"), where $\vec{\mu}_{p}^{r}$ is the set of messages $\left\langle\right.$ vote $_{p}$, ts $_{p}$, histor $\left.y_{p},-\right\rangle$ received in the selection round. When a value $v$ is locked, no value $v^{\prime} \neq v$ can be returned by $F L V\left(\vec{\mu}_{p}^{r}\right)$. On the other hand, if any value can be selected, then $F L V\left(\vec{\mu}_{p}^{r}\right)$ may return ?. If no enough information is provided to $F L V\left(\vec{\mu}_{p}^{r}\right)$ (which may occur, for instance, if a validator does not receive any message during a selection round), then $n u l l$ is returned. A formal definition of $F L V\left(\vec{\mu}_{p}^{r}\right)$ can be found in Section 3.2.

The selection round is executed as follows. Each process $p$ first sends its state and the set $S$ of processes output by function Selector $(p, \phi)$ to the processes in $S$ (line 7 ). Based on the set of messages received, each honest process selects a value (line 9). If any value can be selected (i.e., $F L V\left(\vec{\mu}_{p}^{r}\right)$ returns ?), the selected value is deterministically chosen among $\vec{\mu}_{p}^{r}$ (lines 10-11). When a value has been selected (i.e., selected $p \neq n u l l$ ), process $p$ sets its vote to the selected value, and $\operatorname{logs}$ the selected value in the history (lines 12-14). At the end of the selection round the set of validators for the next round is elected. Line 15 guarantees

\footnotetext{
${ }^{5}$ Bounding the size of the variable histor $_{p}$ requires an additional round of communication. More details can be found in [3].
} 


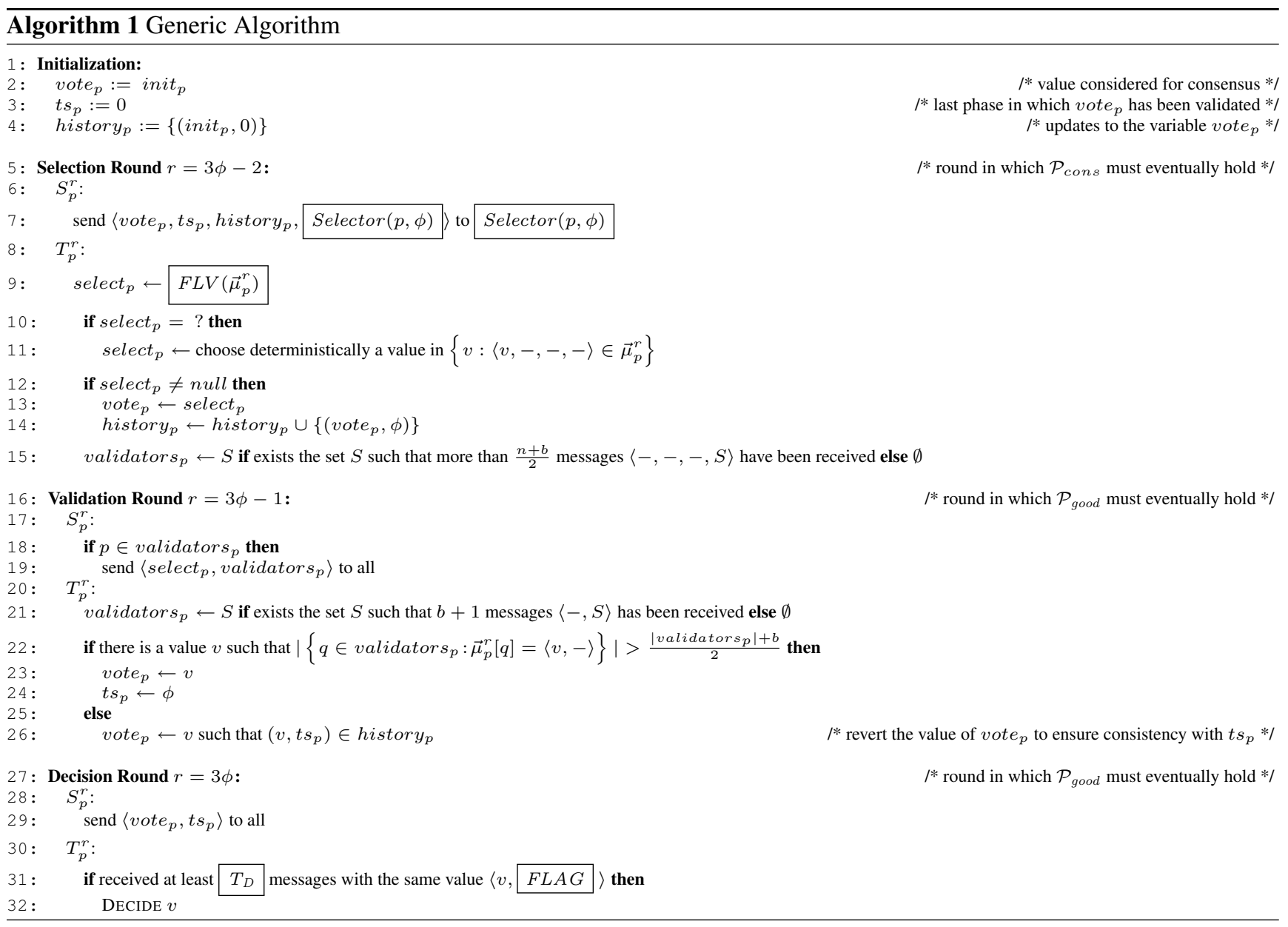

that all honest processes that consider a non-empty set of validators, have the same set of validators.

For termination, the selection round must ensure that all correct validators have selected the same value. This is ensured whenever $\mathcal{P}_{\text {cons }}$ holds, ${ }^{6}$ since all correct processes execute the function $F L V\left(\vec{\mu}_{p}^{r}\right)$ on the same set of messages.

Optimization: The selection round can be suppressed in the first phase. This requires to initialize the variable selected $_{p}$ with init $_{p}$, and validator $s_{p}$ with the same set $S$ on each process $p$. Note that it is safe to select $i_{n i t}$ at the first round for the following reason. If no value is initially locked, then any value may be selected by honest selectors. If some value $v$ is initially locked, then by definition all honest selectors have $i n i t_{p}=v$, and all honest selectors select $v$.

The Validation Round $(r=3 \phi-1)$. The role of this round is for every honest process $p$ to determine which value selected by validators in the selection round is a valid value. Among all honest processes, at most one value may be con-

\footnotetext{
${ }^{6} \mathcal{P}_{\text {cons }}$ is defined in [17] for rounds in which all processes send to all processes. We assume here variant of $\mathcal{P}_{\text {cons }}$ that does not require all-to-all message exchange. The adaptation is trivial.
}

sidered to be valid.

The validation round is executed as follows. Each validator first sends the value selected in the selection round together with the set of validators (line 19). Then, each honest process $p$ computes the set of validators as follows: validator $s_{p}$ is the set $S$ such that $p$ receives $b+1$ messages $\langle-, S\rangle$, or $\emptyset$ if no set satisfies this condition (line 21). Based on this set, each process tries to determine a valid value $v$. If it observes that a majority of correct validators have selected the same value $v$, then $v$ is a valid value. In that case a process $p$ sets its vote $v_{\text {ote }}$ to $v$, and updates its timestamp $t s_{p}$ to the current phase $\phi$ (lines 22-24). Otherwise, the vote is reverted to the value corresponding to $t s_{p}$ (line 26). ${ }^{7}$

Optimizations: If the function Selector $(p, \phi)$ returns the same set of processes on each process $p$ and in all phases $\phi$, then validator $s_{p}$ at line 15 can be set to $\operatorname{Selector}(p, \phi)$, and line 21 can be suppressed. As a result, the sets Selector $(p, \phi)$ and validator $_{p}$ do not need to be sent respectively at lines 7 and 19 .

\footnotetext{
${ }^{7}$ Line 26 is not mandatory, but it allows us to simplify the instantiation of function $F L V\left(\vec{\mu}_{p}^{r}\right)$.
} 
The same holds with benign faults when $|\operatorname{Selector}(p, \phi)|=1$ on each process $p$ and in all phases $\phi$ : validator $s_{p}$ at line 21 is the process from which the validation round message is received.

The Decision Round $(r=3 \phi)$. The decision round determines the conditions that must hold for a process to decide. Concretely, each process starts by sending its vote and its timestamp (line 29). A process then decides if it receives a threshold number $T_{D}$ of identical votes which satisfy some criteria defined by the flag FLAG. To our knowledge, only two criteria have been considered in literature: (1) $F L A G=\phi$ : only votes that have been validated in the current phase $\phi$ are considered, and (2) FLAG $=*$ : all votes are considered. In the latter case, the validation round can be suppressed. As a consequence, variables $t s_{p}$ and history $y_{p}$ are no more necessary. Moreover, the set Selector $(p, \phi)$ does not need to be sent at line 7, and line 15 can be suppressed.

Optimization: The decision round of phase $\phi$ can be executed concurrently with the selection round of phase $\phi+1$.

\subsection{Parameters}

We identify two categories of parameters. The first category is related to the decision round, and contains the parameters $T_{D}$ and FLAG. As shown in Section 4, these two parameters influence the properties of the instantiated algorithm (i.e., $n$, process state, and number of rounds per phase). The second category contains the functions Selector $(p, \phi)$ and $F L V\left(\vec{\mu}_{p}^{r}\right)$ which define the selection and the validation rounds.

FLAG: The parameter FLAG defines which votes are taken into account in the decision round: all votes (if $F L A G=*$ ), or only the votes that are valid in the current phase (if $F L A G=\phi$ ). In the former case, the validation round can be suppressed.

$T_{D}$ : The parameter $T_{D}$ defines the number of identical votes that is required to decide. To ensure termination, the votes of faulty (honest) and Byzantine processes must not be required to decide. Hence, $T_{D} \leq n-b-f$.

Selector $(p, \phi)$ : The function Selector $(p, \phi)^{8}$ returns a set of processes $S \subseteq \Pi$ that represents $p$ 's suggestion for the set validators in phase $\phi$. It must satisfy the following two properties:

- Selector-validity: If $\mid$ Selector $(p, \phi) \mid>0$, then $\mid$ Selector $(p, \phi) \mid>b$.

- Selector-liveness: There exists a good phase $\phi_{0}$ such that:

SL1: $\forall p, q \in \mathcal{C}:$ Selector $\left(p, \phi_{0}\right)=\operatorname{Selector}\left(q, \phi_{0}\right)$,

\footnotetext{
${ }^{8}$ Selector is not really a function. It is rather a problem defined by properties. However, calling it a function is somehow more intuitive. The same comment applies to $F L V$.
}

\begin{abstract}
SL2: if $F L A G=*$, then $\left|\operatorname{Selector}\left(p, \phi_{0}\right) \cap \mathcal{C}\right| \geq T_{D}$,
SL3: if $F L A G=\phi$, then $\mid$ Selector $\left(p, \phi_{0}\right) \cap \mathcal{C} \mid>$ $\frac{\left|\operatorname{Selector}\left(p, \phi_{0}\right)\right|+b}{2}$.
\end{abstract}

$F L V\left(\vec{\mu}_{p}^{r}\right)$ : The function $F L V\left(\vec{\mu}_{p}^{r}\right)$ must satisfy the following three properties:

- FLV-validity: If $F L V\left(\vec{\mu}_{p}^{r}\right)$ returns $v$ such that $v \neq$ ? and $v \neq n u l l$, then $v \in\left\{\right.$ vote $:\langle$ vote,,--$\left.\rangle \in \vec{\mu}_{p}^{r}\right\}$.

- FLV-agreement: If value $v$ is locked in round $r$, only $v$ or $n u l l$ can be returned.

- FLV-liveness: If $\forall q \in \mathcal{C}: \vec{\mu}_{p}^{r}[q] \neq \perp$, then null cannot be returned.

\subsection{Correctness of the Generic Algorithm}

Correctness of our generic alogrithm is based on the following two lemmas from which Theorem 1 can be proved. All proofs can be found in [19].

We now prove that the generic algorithm solves consensus. Our proof is based on four lemmas.

Lemma 1. If Selector-validity holds, then the following property holds on every honest process $h$ and in every phase $\phi:$ at line 21, if $\mid$ validator $s_{h} \mid>0$ then $\mid$ validator $s_{h} \mid>b$.

Proof. Assume for the contradiction that an honest process $p$ has validator $s_{p}=S \neq \emptyset$ at line 21 where $|S| \leq b$. Since at most $b$ processes are Byzantine, this means that an honest processes $\hat{p}$ sent $\langle-, S\rangle$ at line 19 . By line 15 , more than $\frac{n+b}{2}-b$ honest processes send message $\langle-,-,-, S\rangle$ to $\hat{p}$. Since by definition $n \geq b$, it follows that at least one honest process $h$ sent $\langle-,-,-, S\rangle$ to $\hat{p}$ in round $3 \phi-2$. A contradiction with Selector-validity and the fact that $h$ is an honest process.

Lemma 2. If Selector-validity holds, then the following property holds on every honest process $h$ and in every phase $\phi$ : if process $h$ set vote ${ }_{h}$ to $v$ and $t s_{h}$ to $\phi$ at lines 2324 , then at least one honest process has sent $\langle v,-\rangle$ at line 19.

Proof. Assume that a process $h$ set vote $_{h}$ to $v$ and $t s_{h}$ to $\phi$ at lines 23-24. Therefore, validator $_{h}$ is non empty at line 21 in phase $\phi$. By Lemma 1, we have validator $_{h}>b$ and $\frac{\text { validator } s_{h}+b}{2}>b$. Therefore, condition at line 22 can only be true for $v$ if an honest process has sent $\langle v,-\rangle$ at line 19.

Lemma 3. In every phase $\phi$, we have for each pair of honest processes $p$ and $q$ : if validator $s_{p} \neq \emptyset$ and validator $_{q} \neq \emptyset$ at line 21 , then validator $s_{p}=$ validator $s_{q}$. 
Proof. Assume for a contradiction that in some phase $\phi_{0}$ two honest processes $p$ and $q$ have respectively validator $s_{p}=S \neq \emptyset$ and validator $s_{q}=S^{\prime} \neq \emptyset$ at line 21 with $S \neq S^{\prime}$. Since at most $b$ processes are Byzantine, this means that two honest processes $\hat{p}$ and $\hat{q}$ respectively sent $\langle-, S\rangle$ and $\left\langle-, S^{\prime}\right\rangle$ at line 19. By line 15 , more than $\frac{n+b}{2}-b$ honest processes send message $\langle-,-,-, S\rangle$ to $\hat{p}$ and more than $\frac{n+b}{2}-b$ honest processes send message $\left\langle-,-,-, S^{\prime}\right\rangle$ to $\hat{q}$ in round $3 \phi_{0}-2$. Since by definition $n \geq b$, it follows that at least one honest process $h$ sent $\langle-,-,-, S\rangle$ to $\hat{p}$ and $\left\langle-,-,-, S^{\prime}\right\rangle$ to $\hat{q}$. A contradiction with the fact that $h$ is an honest process.

Lemma 4. In every phase $\phi$, if (i) Selector-validity holds, (ii) an honest process $p$ updates vote $e_{p}$ to $v$ and $t s_{p}$ to $\phi$, and (iii) another honest process $q$ updates vote ${ }_{q}$ to $v^{\prime}$ and $t s_{q}$ to $\phi$ (lines 23-24), then $v=v^{\prime}$.

Proof. Assume for a contradiction that in some phase $\phi_{0}$ two honest processes $p$ and $q$ have respectively vote $_{p}=v$ and $t s_{p}=\phi_{0}$, and vote $_{q}=v^{\prime}$ and $t s_{q}=\phi_{0}$. This means that in round $3 \phi_{0}-1$ at least $x-b$ honest processes $(x>$ $\frac{\mid \text { validator } s_{p} \mid+b}{2}$ ) send message $\langle v,-\rangle$ and at least $y-b$ honest processes $\left(y>\frac{\mid \text { validator } s_{q} \mid+b}{2}\right)$ send message $\left\langle v^{\prime},-\right\rangle$. By Lemma 3 we have that validator $s_{p}=$ validator $s_{q}$. Therefore, $x-b+y-b>$ validator $s_{p}-b$. By Selectorvalidity and Lemma 1 , it follows that at least one honest process $h$ sent $\langle v,-\rangle$ to one process and $\left\langle v^{\prime},-\right\rangle$ to another process. A contradiction with the fact that $h$ is an honest process.

Theorem 1. If $(i)$ function $F L V\left(\vec{\mu}_{p}^{r}\right)$ satisfies $F L V$-validity and FLV-agreement, (ii) function Selector $(p, \phi)$ satisfies Selector-validity, (iii-a) FLAG $=\phi$ and $T_{D}>b$ or (iiib) $F L A G=*$ and $T_{D}>\frac{n+b}{2}$, then Algorithm 1 ensures validity, unanimity and agreement.

Termination holds if (iv) $T_{D} \leq n-b-f$, (v) function $F L V\left(\vec{\mu}_{p}^{r}\right)$ satisfies FLV-liveness, and (vi) there is a good phase $\phi_{0}$ in which Selector-liveness holds (SL1, SL2, SL3).

\section{Proof.}

Agreement: Assume for a contradiction that process $p$ decides $v$ in round $r=3 \phi$, and process $p^{\prime}$ decides $v^{\prime} \neq v$ in round $r^{\prime}=3 \phi^{\prime}$. We consider the following two cases for line 31: $F L A G=*$ and $F L A G=\phi$.

$F L A G=*$ : This means that at least $T_{D}$ processes (at least $T_{D}-b$ honest) sent $\langle v$,$\rangle in round r=3 \phi$, and at least $T_{D}$ processes (at least $T_{D}-b$ honest) sent $\left\langle v^{\prime},\right\rangle$ in round $r^{\prime}=3 \phi^{\prime}(*)$. We have two cases to consider: $\phi=\phi^{\prime}$ and $\phi>\phi^{\prime}$.

- $\phi=\phi^{\prime}$ : By (*), $T_{D}-b$ honest processes sent $\langle v$,$\rangle and$ $T_{D}-b$ honest processes sent $\left\langle v^{\prime},\right\rangle$ in round $r=3 \phi$. From (iii-b), $\left(T_{D}-b\right)+\left(T_{D}-b\right)>n-b$. It follows that one honest process $h$ sent $\langle v$,$\rangle to one process and \left\langle v^{\prime},\right\rangle$ to another process. A contradiction with the fact that $h$ is an honest process.

- $\phi^{\prime}>\phi$ : Let $\phi^{\prime}$ be the smallest phase $>\phi$ in which some honest process decides $v^{\prime} \neq v$. By definition of a locked value, $v$ is locked in all phases $>\phi$. Together with the $F L V$-agreement property, no honest process updates its vote with a value $\hat{v} \neq v$ in the selection round of a phase $>\phi$. Since there is no validation round (i.e., FLAG =*), no honest process updates its vote with a value $\hat{v} \neq v$ after phase $\phi$. Together with $(*), T_{D}-b$ honest processes sent $\langle v$,$\rangle , and T_{D}-b$ honest processes sent $\left\langle v^{\prime},\right\rangle$ in round $r^{\prime}=3 \phi^{\prime}$. From (iii-b), $\left(T_{D}-b\right)+\left(T_{D}-b\right)>n-b$. It follows that one honest process $h$ sent $\langle v$,$\rangle to one process$ and $\left\langle v^{\prime},\right\rangle$ to another process. A contradiction with the fact that $h$ is an honest process.

$F L A G=\phi$ : This means that at least $T_{D}$ processes (at least $T_{D}-b$ honest) sent $\langle v, \phi\rangle$ in round $r=3 \phi$, and at least $T_{D}$ processes (at least $T_{D}-b$ honest) sent $\left\langle v^{\prime}, \phi^{\prime}\right\rangle$ in round $r^{\prime}=3 \phi^{\prime}(* *)$. We have two cases to consider: $\phi=\phi^{\prime}$ and $\phi>\phi^{\prime}$.

- $\phi=\phi^{\prime}$ : By (**), $T_{D}-b$ honest processes sent $\langle v, \phi\rangle$ and $T_{D}-b$ honest processes sent $\left\langle v^{\prime}, \phi\right\rangle$. From (iii-a), there is an honest process $h$ that validates $v$ (set vote $h_{h}$ to $v$ and $t s_{h}$ to $\phi$ ) and an honest process $h^{\prime}$ that validates $v^{\prime}$ (set vote ${ }_{h^{\prime}}$ to $v^{\prime}$ and $t s_{h^{\prime}}$ to $\phi$ ) at lines 23-24 of round $\hat{r}=3 \phi-1$. A contradiction with Lemma 4 and (ii).

- $\phi^{\prime}>\phi$ : Let $\phi^{\prime}$ be the smallest phase $>\phi$ in which some honest process decides $v^{\prime} \neq v$. By definition of a locked value, $v$ is locked in all phases $>\phi$. By (**), $T_{D}-b$ honest processes sent $\left\langle v^{\prime}, \phi\right\rangle$. From (iii-a), there is an honest process $h$ that validates $v^{\prime}$ (set $v^{\prime} t_{h^{\prime}}$ to $v^{\prime}$ and $t s_{h^{\prime}}$ to $\phi$ ) at lines 23-24 of round $\hat{r}^{\prime}=3 \phi^{\prime}-1$. By (ii) and Lemma 2, there is an honest process $h^{\prime}$ that sent $\left\langle v^{\prime},-\right\rangle$ at line 19. Therefore, the function $F L V\left(\vec{\mu}_{p}^{r}\right)$ returns $v^{\prime}$ or ? at line 9 on process $h^{\prime}$. A contradiction with the $F L V$-agreement property and the fact that $v$ is locked.

Validity: Follows from the $F L V$-validity property and the assumption that all processes are honest.

Unanimity: Unanimity follows from Lemma 2 together with (ii), the $F L V$-agreement property, (iii-a) and (iii-b).

Termination: Let $\phi_{0}$ be the good phase in which Selector-liveness holds. Let us call selector any process that is in a set Selector $(p, \phi)\left(p, \phi_{0}\right)$ where $p$ is a correct process. By $\mathcal{P}_{\text {cons }}\left(3 \phi_{0}-2\right)$ and Selector-liveness SL1, all correct selectors receive the same set of messages in round $3 \phi_{0}-2$ and therefore select the same value. By $F L V$ liveness and $\mathcal{P}_{\text {cons }}\left(3 \phi_{0}-2\right)$, the value selected cannot be null. Thus, at the end of round $r=3 \phi_{0}-2$, all correct selectors have the same value for select $_{p}$, and vote $_{p}(* * *)$. Let denote this value with $v$. We have two cases to consider: 
$F L A G=*$ and $F L A G=\phi$.

$F L A G=*$ : The validation $3 \phi_{0}-1$ round is skipped. Together with $(* * *)$, all correct selectors send the same message $\langle v,-\rangle$ at line 29. By Selector-liveness SL2 and $\mathcal{P}_{\text {good }}\left(3 \phi_{0}\right)$, all correct processes receives at least $T_{D}$ messages $\langle v,-\rangle$ in round $r=3 \phi_{0}$, and therefore decide.

$F L A G=\phi:$ By Selector-liveness SL1 and $\mathcal{P}_{\text {cons }}$, all correct selectors consider the same set validators at line 15. By Selector-liveness SL3, the set validator contains more than $\frac{\mid \text { validator } \mid+b}{2}$ correct processes (****). Since $F L A G=\phi$, the validation round $3 \phi_{0}-1$ is executed. Together with $(* * * *), \mathcal{P}_{\text {good }}\left(3 \phi_{0}-1\right)$ and lines $22-$ 24 , all correct processes update vote ${ }_{p}$ to $v$ and $t s_{p}$ to $\phi_{0}$. By $\mathcal{P}_{\text {good }}\left(3 \phi_{0}\right)$ and (iv), all correct processes decide in round $3 \phi_{0}$.

\section{Instantiations of the Parameters and Clas- sification}

We present now instantiations of the functions $F L V$ and Selector. We identify three instantiations of $F L V$ function. The first instantiation uses only the variable vote $_{p}$, the second uses the variables vote $_{p}$ and $t s_{p}$, and the last one uses all three variables vote $_{p}, t s_{p}$ and histor $y_{p}$. This leads to three classes of consensus algorithms, as shown in Table 1. Algorithms that belong to the same class have the same values for the parameters $F L A G$ and $T_{D}$. Therefore algorithms from the same class have the same constraint on $n$ (follows from $n \geq T_{D}+b+f$ ) and have the same number of rounds (follows from the value of FLAG, see Section 3.2).

One can observe a tradeoff among these three classes. For instance, when $F L A G=*, T_{D}>\frac{n+3 b+f}{2}$, only two rounds per phase are needed and the process state is the smallest, but it requires the largest $n(n>5 b+3 f)$. The "Examples" column of Table 1 shows which known algorithms correspond to a given class. These examples are discussed in Section 5.

We can make the following comments. First, if $b=0$ (benign faults), classes 2 and 3 are identical, since history can be ignored with benign faults. Therefore Paxos and $\mathrm{CT}^{9}$, which belong to class 2 , also trivially belong to class 3 , case $b=0$. Second, to the best of our knowledge, no existing algorithm corresponds to the class 2 for the case $f=0$ (Byzantine faults). We call this new algorithm MQB (Masking Quorum Byzantine consensus algorithm). ${ }^{10}$ Fi-

\footnotetext{
${ }^{9} \mathrm{CT}$ refers to the Chandra-Toueg consensus algorithm with the failure detector $\diamond \mathcal{S}$.

${ }^{10}$ The quorums used in this algorithm satisfy the property of masking quorums [15]. Note that with respect to the definitions in [15], algorithms of class 1 use opaque quorums, and algorithms of class 3 use dissemination quorums.
}

nally, Table 1 shows that despite its name, the FaB Paxos algorithm does not belong to the same class as the Paxos algorithm.

We now present the three instantiations of the $F L V$ function that lead to the three classes of consensus algorithms. Instantiations of the Selector function are discussed later.

\subsection{Instantiations of $F L V\left(\vec{\mu}_{p}^{r}\right)$}

We give here the intuition of the instantiations. The proofs that the properties defined in Section 3.2 hold can be found in [19].

\subsection{1 $F L V\left(\vec{\mu}_{p}^{r}\right)$ for class 1}

We start the discussion with the $F L V$ function for class 1 (FLAG $=*$ and $T_{D}>\frac{n+3 b+f}{2}$ ), see Algorithm 2 .

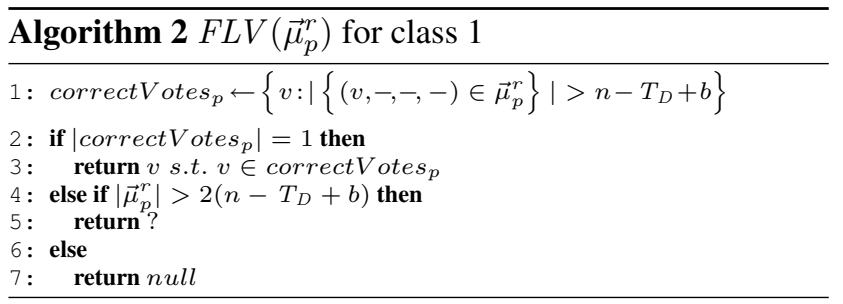

Line 1 is for $F L V$-agreement. We now explain its role with a simple example. Let $v_{1}$ be locked in round $r$. For simplicity, let us reason only on the following case: some honest process $p$ has decided $v_{1}$ in round $r-1$. By Algorithm $1, p$ has received in the decision round $r-1$ at least $T_{D}$ votes $v_{1}$. At least $T_{D}-b$ votes $v_{1}$ are from honest processes, i.e., at most $n-\left(T_{D}-b\right)$ processes have vote $_{p}$ equal to $v_{2} \neq v_{1}(*)$. Therefore, the condition of line 1 can only hold for $v_{1}$, i.e., among the values different from ? and $n u l l, F L V$ can only return $v_{1}$. For $F L V$-agreement to hold, Algorithm 3 must also prevent ? to be returned when $v_{1}$ is locked. The condition of line 4 ensures this. Here is why. Assume that the condition of line 4 holds. This means that $\vec{\mu}_{p}^{r}$ contains more than $2\left(n-T_{D}+b\right)$ messages. With (*), any set of more than $2\left(n-T_{D}+b\right)$ messages contains more than $n-T_{D}+b$ messages equal to $v_{1}$ (this is illustrated in Figure 1 with the case $n=6, b=1, f=0$ and $T_{D}=5$ ). By line 1 , we have $v_{1} \in$ correctVotes $_{p}$, and as explained above, only $v_{1}$ can be in correctVotes $s_{p}$. Therefore, the condition of line 2 holds: Algorithm 2 cannot return? when $v_{1}$ is locked.

Property $F L V$-liveness is ensured by lines 4,5 . This is because when $T_{D}>\frac{n+3 b+f}{2}$, we have $n-b-f>$ $2\left(n-T_{D}+b\right)$. Therefore, receiving a message from all correct processes (i.e., $\left|\vec{\mu}_{p}^{r}\right| \geq n-b-f$ ) implies that the condition of line 4 holds. Property $F L V$-validity is ensured by lines 1-3. 
Table 1. The three classes of consensus algorithms.

\begin{tabular}{|c||c|c||c|c|c||c|}
\hline & $F L A G$ & $T_{D}$ & $n$ & Process state & $\begin{array}{c}\text { Rounds } \\
\text { per phase }\end{array}$ & Examples \\
\hline 1 & $*$ & $>\frac{n+3 b+f}{2}$ & $>5 b+3 f$ & $\left(\right.$ vote $\left._{p}\right)$ & 2 & $\begin{array}{c}\text { OneThirdRule [6] }(b=0) \\
\text { FaB Paxos }[16](f=0)\end{array}$ \\
\hline 2 & $\phi$ & $>3 b+f$ & $>4 b+2 f$ & (vote $\left._{p}, t s_{p}\right)$ & 3 & $\begin{array}{c}\text { Paxos }[11], \text { CT [5] }(b=0) \\
\text { MQB }(f=0) \\
(\text { new alg })\end{array}$ \\
\hline 3 & $\phi$ & $>2 b+f$ & $>3 b+2 f$ & (vote $_{p}$, ts $_{p}$, history $\left.y_{p}\right)$ & 3 & $\begin{array}{c}\text { (Paxos, CT) }(b=0) \\
\text { PBFT }[4](f=0)\end{array}$ \\
\hline
\end{tabular}

\begin{tabular}{|c|c|c|c|c|c|}
\hline \multicolumn{4}{|c|}{$T_{D}-b$} & \multicolumn{2}{|c|}{$\mathrm{n}-\mathrm{T}_{\mathrm{D}}+\mathrm{b}$} \\
\hline $\mathrm{V}_{1}$ & $\mathrm{~V}_{1}$ & $\mathrm{~V}_{1}$ & $\mathrm{~V}_{1}$ & $\mathrm{~V}_{2}$ & $\mathrm{~V}_{2}$ \\
\hline & 1 & \multicolumn{2}{|c|}{$\mathrm{n}-\mathrm{T}_{\mathrm{D}}+\mathrm{b}$} & & Byz. \\
\hline
\end{tabular}

Figure 1. Illustration for $F L V$ for class 1 ( $n=6$, $b=1, f=0, T_{D}=5$ )

Theorem 2. If FLAG $=-$, then Algorithm 2 ensures $F L V$-validity and FLV-agreement. FLV-liveness holds if $T_{D}>\frac{n+3 b+f}{2}$.

Proof.

$F L V$-validity: $F L V$-validity follows from lines 1-3.

$F L V$-agreement: Let $r=3 \phi-2$ be the smallest selection round in which value $v$ is locked. By definition of a locked value, we have two cases to consider (1) all honest processes have vote $_{p}=v$ and unanimity must be ensured (and $r=1$ ), or (2) $v$ has been decided in round $r^{\prime}=3 \phi-3$. We now show that for both cases at least $T_{D}-b$ honest processes sent $\langle v,-,-,-\rangle$ in round $r(*)$.

Case 1: Trivially follows from initialization and $T_{D} \leq$ $n-b-f$.

Case 2: By Algorithm 1, at least $T_{D}$ processes send $\langle v,-\rangle$ in round $r^{\prime}$. Therefore, at least $T_{D}-b$ honest processes have their vote set to $v$, and send $\langle v,-,-,-\rangle$ in round $r$.

We now show that when property $(*)$ holds, Algorithm 2 ensures $F L V$-agreement. Assume for the contradiction that a non null value $v^{\prime} \neq v$ is returned. Two cases must be considered.

$v^{\prime}$ is returned at line 3: Because correctVotes $s_{p}$ is not empty, the set $\vec{\mu}_{p}^{r}$ contains more than $n-T_{D}+b$ messages $\left\langle v^{\prime},-,-,-\right\rangle$. A contradiction with $(*)$.
$?$ is returned at line 5: This means that $\vec{\mu}_{p}^{r}$ contains more than $2\left(n-T_{D}+b\right)$ messages. By $(*), \vec{\mu}_{p}^{r}$ contains at most $n-T_{D}+b$ messages $\left\langle v^{\prime} \neq v,-,-,-\right\rangle$, and therefore, more than $n-T_{D}+b$ messages $\langle v,-,-,-\rangle$. By line 1 , the set correctVotes $s_{p}$ is not empty. By lines 2-3, value $v$ is returned. A contradiction.

This shows that Algorithm 2 ensures $F L V$-agreement in round $r$. Therefore, no honest process $p$ updates its variable vote $_{p}$ and select $t_{p}$ to a value $v^{\prime} \neq v$ in selection round $r$. Because $F L A G=-$, the validation round is skipped. Therefore, property $(*)$ holds in selection round $r^{\prime \prime}=3 \phi+1$. With similar arguments as above, we can show that Algorithm 2 ensures FLV-agreement in round $r^{\prime \prime}$. By a simple induction on $\phi$, we can show that Algorithm 2 ensures $F L V$-agreement in all rounds.

FLV-liveness: Property FLV-liveness is ensured by lines 4-5. This is because when $T_{D}>\frac{n+3 b+f}{2}$, we have $n-b-f>2\left(n-T_{D}+b\right)$. Therefore, receiving a message from all correct processes (i.e., $\left|\vec{\mu}_{p}^{r}\right| \geq n-b-f$ ) implies that the condition of line 4 holds.

\subsection{2 $F L V\left(\vec{\mu}_{p}^{r}\right)$ for class 2}

The $F L V$ function for class $2\left(F L A G=\phi\right.$ and $T_{D}>$ $3 b+f)$ is shown in Algorithm 3, where $\{\# \ldots \#\}$ at line 1 denotes a multiset. Note that when $T_{D} \leq \frac{n+3 b+f}{2}$ which can be the case for instantiations of classes 2 and 3, detecting the locked value only based on votes, as done by Algorithm 2, does not work. Therefore, an additional mechanism is needed: timestamps.

Lines 1 and 2 are for $F L V$-agreement. We now explain their role with a simple example. Let $v_{1}$ be locked in round $r$ that belongs to phase $\phi_{1}+1$. For simplicity, let us reason only on the following case: some honest process $p$ has decided $v_{1}$ in round $r-1$ that belongs to phase $\phi_{1}$. By Algorithm 1, $p$ has received $T_{D}$ messages $\left\langle v_{1}, \phi_{1}\right\rangle$ in the decision round $r-1$. Therefore, at least $T_{D}-b$ honest processes have vote $_{p}=v_{1}$ and $t s_{p}=\phi_{1}$, i.e., at most $n-T_{D}$ honest processes have vote $_{p}=v_{2} \neq v_{1}\left({ }^{*}\right)$. 


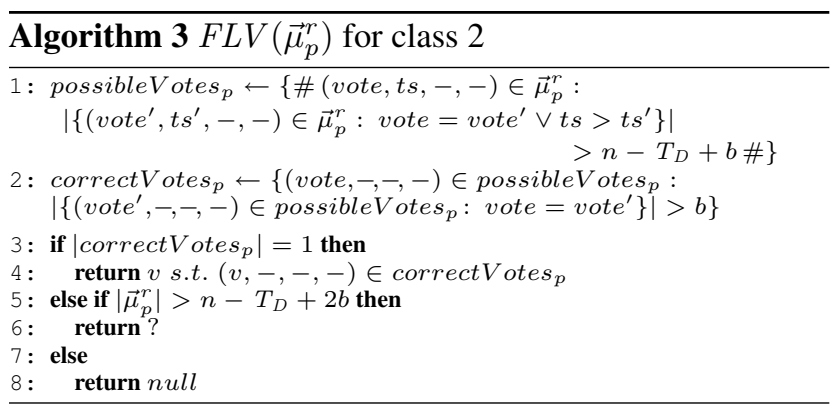

Because only one value can be validated by honest processes in phase $\phi_{1}$ (see Lemma 4), all honest processes with vote $_{p}=v_{2} \neq v_{1}$ have $t s_{p}<\phi_{1}$. It follows that for every honest process $p$, we have vote $_{p}=v_{1}$ or $t s_{p}<\phi_{1}(* *)$. Together with (*), no message $\left\langle v_{2} \neq v_{1},-,-,-\right\rangle$ sent by an honest process can satisfy the condition of line 1 . In other words, the set possibleVotes $s_{p}$ may contain at most $b$ messages $\left\langle v_{2} \neq v_{1},-,-,-\right\rangle$, i.e., the messages sent by Byzantine processes. Line 2 prevents such messages to be in correctVotes $s_{p}$. This shows that among the values different from ? and $n u l l$, only $v_{1}$ can be returned.

$$
\begin{array}{|l|l|l|l|l|}
\hline\left(V_{1}, \Phi_{1}\right) & \left(V_{1}, \Phi_{1}\right) & \left(V_{1}, \phi_{1}\right) & \left(V_{2}, \Phi_{2}{ }^{\prime}<\phi_{1}\right) & \left(V_{2}, \Phi_{2}>\phi_{1}\right) \\
\underbrace{\mathrm{T}_{\mathrm{D}}-\mathrm{b}}_{1} & \underbrace{\mathrm{n}-\mathrm{T}_{\mathrm{D}}+\mathrm{b}}_{\mathrm{b}} \text { Byz. } \\
\hline
\end{array}
$$

Figure 2. Illustration for $F L V$ for class 2 ( $n=5$, $b=1, f=0, T_{D}=4$ )

For FLV-agreement to hold, Algorithm 3 must also prevents ? to be returned when $v_{1}$ is locked. The condition of line 5 ensures this. Here is why. Assume that the condition of line 5 holds. This means that $\vec{\mu}_{p}^{r}$ contains more than $n-T_{D}+2 b$ messages. With (*), the set $\vec{\mu}_{p}^{r}$ contains at least $b+1$ messages $\left\langle v_{1}, \phi_{1},-,-\right\rangle$ from honest processes (this is illustrated in Figure 2 for the case $n=5, b=1, f=0$, $\left.T_{D}=4\right)$. With (**) and the fact that $\vec{\mu}_{p}^{r}$ contains more than $n-T_{D}+b$ messages from honest processes (see Figure 2), the $b+1$ messages $\left\langle v_{1}, \phi_{1},-,-\right\rangle$ satisfy the condition of line 1 . By line $2,\left\langle v_{1}, \phi_{1},-,-\right\rangle$ is in correctVotes $s_{p}$. Moreover, as discussed above, only $v_{1}$ can be in correctVotes $s_{p}$. Therefore, the condition of line 3 holds: Algorithm 3 cannot return ? when $v_{1}$ is locked.

Property $F L V$-liveness is ensured by lines 5, 6. This is because when $T_{D}>3 b+f$, we have $n-b-f>$ $n-T_{D}+2 b$. Therefore, receiving a message from all correct processes (i.e., $\left|\vec{\mu}_{p}^{r}\right| \geq n-b-f$ ) ensures that the condition of line 5 holds. Property $F L V$-validity is ensured by lines $1-4$.

Theorem 3. If $F L A G=\phi$ and Selector $(p, \phi)$-validity holds, then Algorithm 3 ensures FLV-validity and FLVagreement. FLV-liveness holds if in addition $T_{D}>3 b+f$.

Proof.

$F L V$-validity: $F L V$-validity follows from lines 1-4.

FLV-agreement: Let $r=3 \phi-2$ be the smallest selection round in which value $v$ is locked. By definition of a locked value, we have two cases to consider (1) all honest processes have vote $_{p}=v$ and unanimity must be ensured (and $r=1$ ), or (2) $v$ has been decided in round $r^{\prime}=3 \phi-3$. We now show that for both cases in round $r$ at least $T_{D}-b$ honest processes sent $\langle v, \hat{\phi} \geq \phi-1,-,-\rangle\left(^{*}\right)$, and for all honest processes $q$, we have vote $_{q}=v \vee t s_{q}<\phi-1$ ) (**).

Case 1: Trivially follows from initialization and $T_{D} \leq$ $n-b-f$.

Case 2: By Algorithm 1, at least $T_{D}$ processes send $\langle v, \phi-1\rangle$ in round $r^{\prime}$. Therefore, at least $T_{D}-b$ honest processes have their vote set to $v$, and send $\langle v, \phi-1,-,-\rangle$ in round $r$ (which shows $(*)$ ). This means that an honest process $p$ updates $v o t e_{p}$ to $v$ and $t s_{p}$ to $\phi$ in the validation round of phase $\phi-1$. By Lemma 4, no honest process update its vote to a value $v^{\prime} \neq v$ in this validation round (which shows $(* *))$.

We now show that when properties (*) and (**) hold, Algorithm 3 ensures FLV-agreement. Assume for the contradiction that a non null value $v^{\prime} \neq v$ is returned. Two cases must be considered.

$v^{\prime}$ is returned at line 4: Because correctVotes $s_{p}$ is not empty, the set possibleVotes ${ }_{p}$ contains more than $b$ messages $\left\langle v^{\prime},-,-\right\rangle$. It follows that an honest process sent a message $\left\langle v^{\prime}, \phi^{\prime},-,-\right\rangle$. By (**), $\phi^{\prime}<\phi-1$. By line 1 , at least $n-T_{D}$ honest processes sent a message $\left\langle v^{\prime \prime}, \phi^{\prime \prime},-,-\right\rangle$ with $v^{\prime \prime}=v^{\prime}$ or $\phi^{\prime \prime}<\phi^{\prime}<\phi-1$. A contradiction with (*).

? is returned at line 6: This means that $\vec{\mu}_{p}^{r}$ contains more than $n-T_{D}+2 b$ messages (and more than $n-T_{D}+b$ messages from honest processes $\left.(* * *)\right)$. By (*), $\vec{\mu}_{p}^{r}$ contains at most $n-T_{D}+b$ messages different from $\langle v, \hat{\phi} \geq \phi-1,-,-\rangle$, and therefore, more than $b$ messages $\langle v, \hat{\phi} \geq \phi-1,-,-\rangle$. By line $1,(* *)$ and (***), the set possibleVotes $s_{p}$ contains more than $b$ messages $\langle v,-,-,-\rangle$. Therefore, the set correctVotes $s_{p}$ contains a message $\langle v,-,-,-\rangle$. By lines $3-4$, value $v$ is returned. A contradiction.

This shows that Algorithm 3 ensures $F L V$-agreement in round $r$. Therefore, no honest process $p$ updates its variable vote $_{p}$ and select $p$ to a value $v^{\prime} \neq v$ in selection round $r$. By Lemma 2, no honest process $p$ updates its 
vote to a value $v^{\prime} \neq v$ in the validation round $r+1$. Therefore, properties $(*)$ and $(* *)$ hold in selection round $r^{\prime \prime}=3 \phi+1$. With similar arguments as above, we can show that Algorithm 3 ensures FLV-agreement in round $r^{\prime \prime}$. By a simple induction on $\phi$, we can show that Algorithm 3 ensures $F L V$-agreement in all rounds.

$F L V$-liveness: Property $F L V$-liveness follows from lines 5 and 6 . This is because when $T_{D}>3 b+f$, we have $n-b-f>n-T_{D}+2 b$. Therefore, receiving messages from all correct processes (i.e., $\vec{\mu}_{p}^{r} \geq n-b-f$ ) ensures that the condition of line 5 holds.

\subsection{3 $F L V\left(\vec{\mu}_{p}^{r}\right)$ for class 3}

The $F L V$ function for class 3 ( $F L A G=\phi$ and $T_{D}>2 b+$ $f$ ) is shown in Algorithm 4. Observe that for instantiations of class $3, T_{D}$ can be $\leq 3 b+f$. Therefore, detecting the locked value only based on votes and timestamps, as done by Algorithm 3, does not work. Therefore, an additional mechanism is needed: the history log.

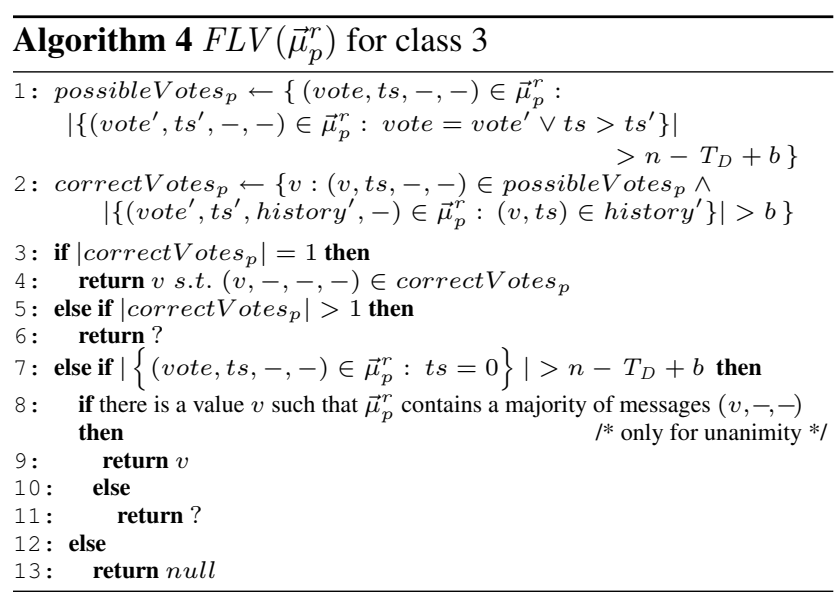

Similarly to Algorithm 3, lines 1 and 2 are for $F L V$ agreement. Their role can be explained with a simple example. Consider that value $v_{1}$ is locked in round $r$ that belongs to phase $\phi_{1}+1$. For simplicity, let us first assume that some honest process $p$ has decided $v_{1}$ in round $r-1$ that belongs to phase $\phi_{1}$. Consider Figure 3. For the same reason as for Algorithm 3, at least $T_{D}-b$ honest processes have vote $_{p}=v_{1}$ and $t s_{p}=\phi_{1}(*)$, i.e., at most $n-T_{D}$ honest processes have vote $_{p}=v_{2} \neq v_{1}$. Furthermore, for every honest process $p$, we have vote $_{p}=v_{1}$ or $t s_{p}<\phi_{1}(* *)$. Together with $(*)$, no message $\left\langle v_{2} \neq v_{1},-,-,-\right\rangle$ sent by an honest process can satisfy the condition of line 1 . Said differently, apart from messages $\left\langle v_{1},-,-,-\right\rangle$, only messages $\left\langle v_{2} \neq v_{1}, \phi_{2},-,-\right\rangle$ sent by Byzantine processes can be in

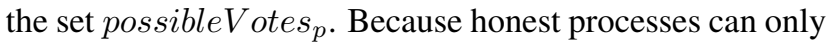
update history at line 14 of Algorithm 1, no honest process has a pair $\left(-, \phi_{2}>\phi_{1}\right)$ in its history in the sending step of round $r$. It follows that only messages $\left\langle v_{1},-,-,-\right\rangle$ can be in correctVotes $s_{p}$ at line 2 . Therefore, when a value $v_{1}$ is locked, lines 1 and 2 prevent any value $v \neq v_{1}$ or $v=$ ? to be returned at lines 4 and 6 . By (*) together with $\phi_{1}>0$, condition of line 7 never holds in our example.

To understand the role of lines 8-11, we have to consider another example. Let all honest processes have initially vote $_{p}=v_{1}$. With the same arguments as above, it follows that no value different from $v_{1}$ or null can be returned at lines 4 and 6 . However, the condition of line 7 might hold. In this case, $\vec{\mu}_{p}^{r}$ contains more than $n-T_{D}$ messages $\left\langle v_{1}, 0,-,-\right\rangle$ from honest processes, and at most $b$ messages $\left\langle v_{2} \neq v_{1}, 0,-,-\right\rangle$ from Byzantine processes. Because $T_{D} \leq n-b-f$, we have $n-T_{D} \geq b$, and $v_{1}$ is returned at line 9 . In other words, line 9 ensures $F L V$ agreement when unanimity is considered.

Let us now discuss $F L V$-liveness. For this property to hold, we need a stronger variant of Selector-validity: ${ }^{11}$

- Selector-strongValidity: If $|\operatorname{Selector}(p, \phi)|>0$, then $|\operatorname{Selector}(p, \phi)|>3 b+2 f$.

This requirements can be explained as follows. Let $\vec{\mu}_{p}^{r}$ contains the messages from all the $n-b-f$ correct processes. There are two cases to consider: (1) correct processes sent only $\langle-, 0,-,-\rangle,(2)$ at least one correct process sent $\langle-, t s>0,-,-\rangle$. Note that $T_{D}>2 b+f$ ensures $n-b-f>n-T_{D}+b(*)$. In case (1), by (*) the condition of line 7 holds, and null cannot be returned at line 13 . In case (2), let $\nu$ denote the subset of messages in $\vec{\mu}_{p}^{r}$ that are from correct processes, and let $t s_{\nu}$ be the highest timestamp in $\nu$. By Lemma 4 and Algorithm 1, there is a unique value $v_{\nu}$ such that $\left\langle v_{\nu}, t s_{\nu},-,-\right\rangle \in \nu$. Together with (*), this ensures that the set possibleVotes $s_{p}$ is not empty, and contains $\left\langle v_{\nu}, t s_{\nu},-,-\right\rangle$. The Selector-strongValidity allows us to get a stronger variant of Lemma 2: it ensures that if process $h$ set vote $_{h}$ to $v$ and $t s_{h}$ to $\phi$ at lines 2324 , then at least $b+1$ correct processes have sent $\langle v,-\rangle$ at line 19. As a result, any correct process that validates $v_{\nu}$ in the validation round $3 t s_{\nu}-1$ received $v_{\nu}$ from at least $b+1$ correct processes. Therefore, at least $b+1$ correct processes have selected $v_{\nu}$ in round $3 t s_{\nu}-2$, and these processes have $\left(v_{\nu}, t s_{\nu}\right)$ is their history. This implies that the set correctVotes $_{p}$ is non empty, and a non-null value is returned at line 4 or 6 .

Theorem 4. If FLAG $=\phi$ and $\operatorname{Selector}(p, \phi)$-validity holds, then Algorithm 4 ensures FLV-validity and FLVagreement. FLV-liveness holds if in addition $T_{D}>2 b+f$ and Selector-strong Validity holds.

\footnotetext{
${ }^{11}$ This stronger variant was not introduced in Section 3.2, since the proof of the generic Algorithm 1 does not require the stronger variant. In the proof of Algorithm 1, the stronger variant is hidden in the $F L V$-liveness property.
} 


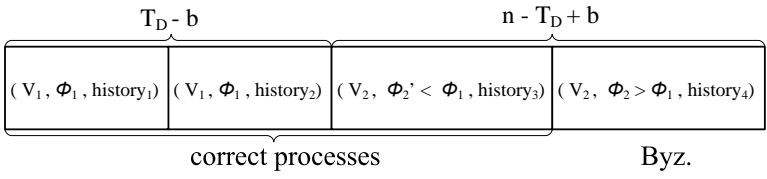

Figure 3. Illustration for $F L V$ for class 3 ( $n=4$, $b=1, f=0, T_{D}=3$ )

Proof.

$F L V$-validity: $F L V$-validity follows from the lines $1-4$ and 8-9.

FLV-agreement: Let $r=3 \phi-2$ be the smallest selection round in which value $v$ is locked. By definition of a locked value, we have two cases to consider (1) all honest processes have vote $_{p}=v$ and unanimity must be ensured (and $r=1$ ), or (2) $v$ has been decided in round $r^{\prime}=3 \phi-3$ (and thus, $\phi-1>1$ ). We now show that for both cases in round $r$ at least $T_{D}-b$ honest processes sent $\langle v, \hat{\phi} \geq \phi-1,-,-\rangle(*)$, for all honest processes $q$, we have $\left(\right.$ vote $\left._{q}=v \vee t s_{q}<\phi-1\right)(* *)$, and for any element (vote,ts) in the set history of $_{q}$ any honest process $q$, we have $\left(\right.$ vote $\left.=v_{1} \vee t s \leq \phi-1\right)(* * *)$. In addition, if less than $T_{D}-b$ honest processes have $t s_{p}>0$, then all honest processes have vote v $_{p}=v(* * * *)$.

Case 1: Trivially follows from initialization and $T_{D} \leq$ $n-b-f$.

Case 2: By Algorithm 1, at least $T_{D}$ processes send $\langle v, \phi-1\rangle$ in round $r^{\prime}$. Therefore, at least $T_{D}-b$ honest processes have their vote set to $v$, and send $\langle v, \phi-1,-,-\rangle$ in round $r$ (which shows $(*)$ ). This means that an honest process $p$ updates vote $_{p}$ to $v$ and $t s_{p}$ to $\phi$ in the validation round of phase $\phi-1$. By Lemma 4 , no honest process update its vote to a value $v^{\prime} \neq v$ in this validation round (which shows $(* *))$.

Property $(* * *)$ trivially follows from the fact that for each honest process the last update of history occured in round $3(\phi-1)-2$. Property (****) trivially follows from $\hat{\phi} \geq \phi-1>1$ and $(*)$, which implies that the precondition of $(* * * *)$ cannot be true.

We now show that when properties $(*),(* *),(* * *)$ and (****) hold, Algorithm 4 ensures FLV-agreement. Assume for the contradiction that a non null value $v^{\prime} \neq v$ is returned. Four cases must be considered.

$v^{\prime}$ is returned at line 4: Because correctVotes $s_{p}$ is not empty, the set $r m s g_{p}^{r}$ contains a message $\left\langle v^{\prime}, \phi^{\prime},-,-\right\rangle$ in possibleVotes $_{p}$ such that an honest process $h$ has $\left(v^{\prime}, \phi^{\prime}\right)$

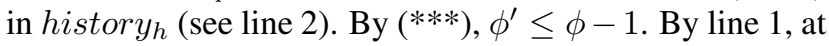
least $n-T_{D}$ honest processes sent a message $\left\langle v^{\prime \prime}, \phi^{\prime \prime},-,-\right\rangle$ with $v^{\prime \prime}=v^{\prime}$ or $\phi^{\prime \prime}<\phi^{\prime} \leq \phi-1$. A contradiction with (*).
$?$ is returned at line 6: Same arguments as the case $v^{\prime}$ is returned at line 4 .

$v^{\prime}$ is returned at line 9: By line 7, the set $\vec{\mu}_{p}^{r}$ contains more than $n-T_{D}+b$ messages $\langle-, 0,-,-\rangle$. Therefore, less than $T_{D}-b$ honest processes has $t s_{p}>0$. By (****), all honest processes has vote $e_{p}=v$. Therefore $\vec{\mu}_{p}^{r}$ contains more than $n-T_{D}$ messages $\langle v, 0,-,-\rangle$ and at most $b$ messages $\left\langle v^{\prime}, 0,-,-\right\rangle$. Because $T_{D} \leq n-b-f$, there is a majority of messages $\langle v, 0,-,-\rangle$ in $\vec{\mu}_{p}^{r}$. A contradiction with line 8 and the fact that $v^{\prime}$ is returned at line 9.

? is returned at line 11: Same arguments as the case $v^{\prime}$ is returned at line 9 .

This shows that Algorithm 4 ensures $F L V$-agreement in round $r$. Therefore, no honest process $p$ updates its variable vote $_{p}$ and select $p$ to a value $v^{\prime} \neq v$ in selection round $r$. Furthermore, no honest process $p$ adds a tuple $\left(v^{\prime} \neq v, \phi\right)$ in selection round $r$. By Lemma 2 , no honest process $p$ updates its vote to a value $v^{\prime} \neq v$ in the validation round $r+1$. Therefore, properties $(*),(* *),(* * *)$ and (****) hold in selection round $r^{\prime \prime}=3 \phi+1$. With similar arguments as above, we can show that Algorithm 4 ensures $F L V$-agreement in round $r^{\prime \prime}$. By a simple induction on $\phi$, we can show that Algorithm 4 ensures $F L V$-agreement in all rounds.

FLV-liveness: Let $\vec{\mu}_{p}^{r}$ contain the messages from all the $n-b-f$ correct processes. There are two cases to consider: (1) correct processes sent only $\langle-, 0,-\rangle$, (2) at least one correct process sent $\langle-, t s>0,-\rangle$. Note that $T_{D}>2 b+f$ ensures $n-b-f>n-T_{D}+b$ (*). $^{*}$ In case (1), by (*) the condition of line 7 holds, and null cannot be returned at line 13 . In case (2), let $S$ denote the subset of messages in $\vec{\mu}_{p}^{r}$ that are from correct processes, and let $t s_{S}$ be the highest timestamp in $S$. By SelectorstrongValidity and Lemma 4 , there is a unique value $v_{S}$ such that $\left\langle v_{S}, t s_{S},-,-\right\rangle \in S$. Together with (*), this ensures that the set possibleVotes $p$ is not empty, and contains $\left\langle v_{S}, t s_{S},-,-\right\rangle$. The Selector-strongValidity allows us to get a stronger variant of Lemma 1 that ensures that $\mid$ validator $_{p} \mid>0$ implies $\mid$ validator $s_{p} \mid>3 b+2 f$. As a result, any correct process that validates $v_{S}$ in the validation round $3 t s_{S}-1$ received $\left\langle v_{S},-\right\rangle$ from more than $\frac{(3 b+2 f)+b}{2}=2 b+f$ processes. Therefore, at least $b+1$ correct processes have selected $v_{S}$ in round $3 t s_{S}-2$, and these processes have $\left(v_{S}, t s_{S}\right)$ in their history. This implies that the set correctVotes ${ }_{p}$ is non empty, and a non-null value is returned at line 4 or 6 .

\subsection{Instantiations of $\operatorname{Selector}(p, \phi)$}

A trivial instantiation of the Selector function consists in always returning the whole set of processes $\Pi$. This trivially satisfies Selector-validity, Selector-strongValidity 
and Selector-liveness. To our knowledge, this instantiation is used in all algorithms for Byzantine faults. However, another possible instantiation can be considered in the Byzantine fault model: it consists in returning the same set $S$ of $b+1$ processes at every process, with $S$ being different in every phase.

In the benign fault model, it is sufficient that the Selector function always returns a single process rather than a set of processes. One such instantiation of the Selector function is the well known rotating coordinator function used in [5]. Another example is the leader election function used in [11].

\section{Instantiation examples}

In this section we show several well-known consensus algorithms obtained from Algorithm 1. Note that because the instantiated algorithms are expressed in the round model, some details of the original algorithms (retransmission rules, leader election, message acceptance policies, etc.) are hidden.

\subsection{Class 1 - OneThirdRule and FaB Paxos}

OneThirdRule [6] The OneThirdRule algorithm, which assumes benign faults only, is obtained from Algorithm 1 with the following parametrization: $T_{D}=\left\lceil\frac{2 n+1}{3}\right\rceil,{ }^{12}$ $F L A G=*$, Selector $(p, \phi)$ returning always $\Pi$ and Algorithm 2 with $T_{D}=\left\lceil\frac{2 n+1}{3}\right\rceil$ as a $F L V$ instantiation. It can be noticed that the instantiation leads to a (small) improvement of the original OneThirdRule algorithm. Details can be found in [19].

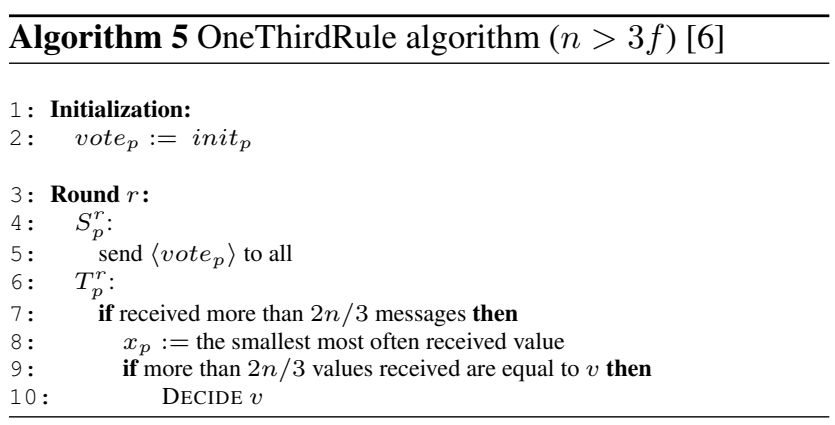

We now compare the instantiated version of the OneThirdRule algorithm with the original algorithm. In the OneThirdRule algorithm, the selection round and the decision round are merged into one single round. Since $T_{D}>2 n / 3$, it is easy to see that the condition for deciding is the same in the two algorithms (compare line 31

\footnotetext{
${ }^{12} T_{D}$ is chosen such that the same number of messages allow the condition at line 31 of Algorithm 1 and the condition at line 4 of Algorithm 2 to hold.
}

of Algorithm 1 and line 9 of Algorithm 5). However, the selection condition of the two algorithms have (minor) differences. Specifically, it is easy to see that whenever some value is selected by Algorithm 5 (lines 7 and 8), then some value (not necessarily the same) is also selected by Algorithm 2. The opposite is not true. If the number of messages received is not larger than $2 n / 3$, Algorithm 5 will not select any value, while Algorithm 2 may still select a value by line 3 . In this sense, the instantiation of Algorithm 1 is a (small) improvement of the OneThirdRule algorithm.

FaB Paxos [16] FaB Paxos algorithm is designed for the Byzantine fault model $(f=0)$ and requires $n>5 b$ to tolerate $b$ Byzantine faults. The algorithm is expressed in the context of "proposers", "acceptors" and "learners". For simplicity, in our framework, consensus algorithms are expressed without considering these roles. We get FaB Paxos algorithm from Algorithm 1 by applying the following parametrization: $T_{D}=\lceil(n+3 b+1) / 2\rceil, F L A G=*$, Selector $(p, \phi)$ returning always $\Pi$, and Algorithm 6 as an instantiation of FLV function (Algorithm 2 with $T_{D}=$ $\lceil(n+3 b+1) / 2\rceil)$.

We now compare the instantiated version of $\mathrm{FaB}$ Paxos with the original algorithm. Since $T_{D}=\lceil(n+3 b+1) / 2\rceil$, it is easy to see that the deciding condition is the same in both algorithms. However, the selection condition of the two algorithms have (minor) differences. With the original FaB Paxos, the selection rule is applied when $n-b$ messages are received. In that case, a value $v$ is selected if it appears at least $\lceil(n-b+1) / 2\rceil$ times in the set of received messages; otherwise any value can be selected. ${ }^{13}$ Therefore, if a number of received messages is smaller than $n-b, \mathrm{FaB}$ Paxos will not select any value, while Algorithm 6 may still select a value by line 3 . In this sense, the instantiation of Algorithm 1 is a (small) improvement of the original FaB Paxos algorithm.

The original FaB Paxos algorithm uses a coordinatorbased implementation of the $\mathcal{P}_{\text {cons }}$ predicate, based on signed messages [17]. By using the coordinator-free and signature-free implementation of $\mathcal{P}_{\text {cons }}$ [2], we can obtain coordinator-free and signature-free variant of FaB Paxos.

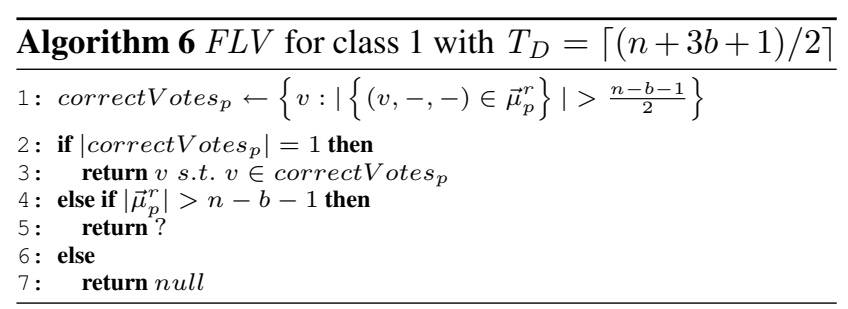

\footnotetext{
${ }^{13}$ Note that the condition at line 1 of Algorithm 6 for selecting a value $v$ requires smaller number of messages to be received than in the original algorithm. For example, when $n=7$ and $b=1$, FaB Paxos requires at least 4 messages equal to $v$ to be received (at least $\lceil(n-b+1) / 2\rceil(=4))$, while Algorithm 6 requires 3 messages (more than $\frac{n-b-1}{2}(=2)$ ).
} 


\subsection{Class 2 - MQB}

MQB is our new Byzantine consensus algorithm that requires $n>4 b$. Compared to PBFT, it has the advantage not to need the (unbounded) variable history $_{p}$, at the cost of requiring $n>4 b$ instead of $n>3 b$ (for PBFT). We get MQB from Algorithm 1 with the following parametrization: ${ }^{14} T_{D}=\left\lceil\frac{n+2 b+1}{2}\right\rceil$, FLAG $=\phi$, Selector $(p, \phi)=\Pi$ and Algorithm 3 with $T_{D}=\left\lceil\frac{n+2 b+1}{2}\right\rceil$ as a FLV instantiation. Depending on the implementation of the $\mathcal{P}_{\text {cons }}$ predicate (coordinator-based or coordinator-free), we get coordinator-based or coordinator-free variants of MQB.

\subsection{Class 3 - Paxos and PBFT}

We discuss Paxos as part of class 3 (rather than as part of class 2) to show the similarities between Paxos and PBFT, namely that the selection round for Paxos and PBFT are derived from the $F L V$ function for class 3. Paxos and PBFT are algorithms that solves a sequence of instances of consensus (state machine replication). We consider here the instantiation of a single instance of consensus that represents the "core" of these algorithms. Both algorithms incorporate the optimizations related to Selector $(p, \phi)$ and validators mentioned in Section 3.1.

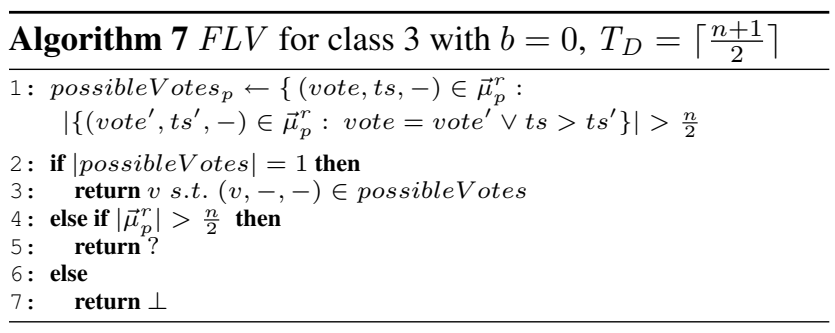

Paxos [11] Paxos assumes benign faults only $(b=0)$ and requires $n>2 f$. We get Paxos from Algorithm 1 with the following parametrization: ${ }^{15} \quad T_{D}=\left\lceil\frac{n+1}{2}\right\rceil, F L A G=\phi$, Selector $(p, \phi)$ implementing leader election, and Algorithm 7 as a $F L V$ instantiation.

With only benign faults, the instantiation of the function $F L V$ can be simplified. We now explain how to get Algorithm 7 from Algorithm 4. First, we can observe that any message $\langle$ vote, ts, history $\rangle$ has the following property: (vote, ts $) \in$ history. Therefore, the set correctVotes $s_{p}$ is

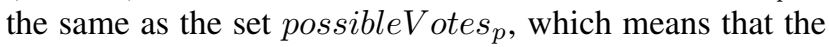
set correctVotes $p$ is not needed. It follows that history is not needed in the $F L V$ function, and by extension, the variable history $_{p}$ is not needed in the consensus algorithm.

Because the unanimity property is not relevant in the benign case, lines 8-9 of Algorithm 4 can be removed. This

\footnotetext{
${ }^{14}$ See footnote 12 , here with reference to line 5 of Algorithm 3 instead of line 4 of Algorithm 2.

${ }^{15}$ See footnote 12 , here with reference to line 7 of Algorithm 4 instead of line 4 of Algorithm 2.
}

allows us to merge lines 5-11 of Algorithm 4 into lines 4-5 of Algorithm 7.

PBFT [4] PBFT is designed for Byzantine faults $(f=0)$ and requires $n>3 b$. We get PBFT from Algorithm 1 with the following parametrization: $T_{D}=2 b+1, F L A G=\phi$, Selector $(p, \phi)=\Pi$ and Algorithm 8 as a $F L V$ instantiation. To get the instantiation as close as possible to PBFT, we have set $n=3 b+1$, as in PBFT.

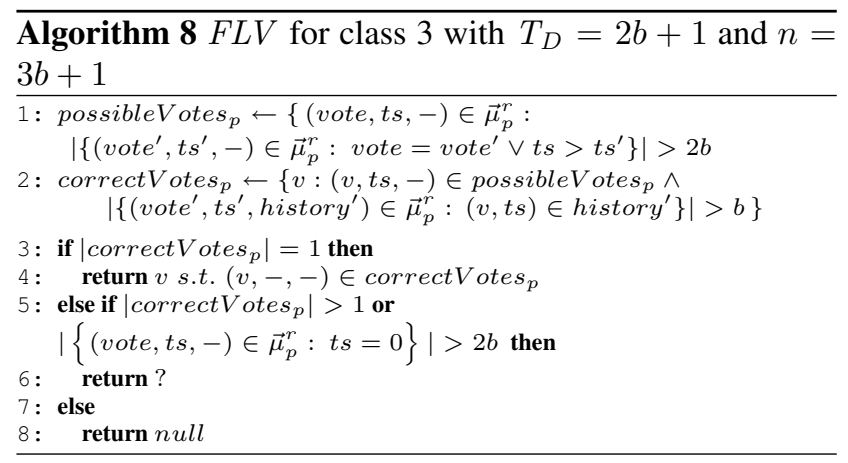

We explain now how to get Algorithm 8 from Algorithm 4. PBFT does not consider the unanimity property, which allows a significant simplification of Algorithm 4. Indeed, without the unanimity property, lines 8-9 of Algorithm 4 can be removed. Then, we can merge the conditions of line 5 and line 7 of Algorithm 4 into line 5 of Algorithm 8.

PBFT uses a coordinator-based implementation of $\mathcal{P}_{\text {cons }}$ predicate that does not require signed messages [17]. By using the coordinator-free implementation of $\mathcal{P}_{\text {cons }}$ [2], we get a coordinator-free variant of PBFT.

\section{Randomized consensus algorithms}

Algorithm 1 can be adapted to support randomized consensus algorithms. The first modification is the introduction of randomization. In the context of binary consensus (initial value 0 or 1 ), line 11 is replaced with " select $_{p}:=1$ or 0 with probability 0.5 ". This allows all correct processes, by repeating the execution of the selection round, to select the same value with probability 1 .

A second modification is needed, which is related to the "reliable channel" assumption of these algorithms. This assumption can be expressed by the following communication predicate that is required to hold in every round $r$ instead of predicates $\mathcal{P}_{\text {cons }}$ and $\mathcal{P}_{\text {good }}$ :

$$
\mathcal{P}_{\text {rel }}(r) \equiv \forall p \in \mathcal{C}:\left|\left\{m \in \vec{\mu}_{p}^{r}: m \neq \perp\right\}\right| \geq n-b-f .
$$

Therefore, randomized protocols need a slightly different $F L V$-liveness property: for any set $\vec{\mu}_{p}^{r}$ with $n-b-f$ messages (instead of any set with all messages from correct 
processes), FLV must return a value different from null. Note that Algorithms 2 and 3 ensure this property, but not Algorithm 4. In other words, we can easily transform any consensus algorithm of class 1 or 2 into a randomized algorithm. We believe that this is not possible for consensus algorithms of class 3 .

The instantiations of Ben-Or's binary consensus algorithms [1] from Algorithm 1 can be found in [19].

Ben-Or [1]. We now show how these adaptations allow us to instantiate the Ben-Or binary consensus algorithms. We get those algorithms from the adapted Algorithm 1 with the following parametrization: $F L A G=\phi$, the function $\operatorname{Selector}(p, \phi)$ that always return $\Pi$ and Algorithm 9 as an instantiation of the $F L V$ function. The version of the Ben-Or algorithm dedicated to benign faults assumes $T_{D}=f+1$ (and $n>2 f$ ), while the version dedicated to Byzantine faults assumes $T_{D}=3 b+1$ (and $n>4 b$ ). Both algorithms implement the optimizations related to selection and decision rounds that are described in Section 3.1.

The Ben-Or algorithms can be considered as algorithms of class 2 in the sense that Algorithm 9 can be seen as a variant of Algorithm 3. We now explain how to get Algorithm 9 from Algorithm 3. Because $n-b-f>n-T_{D}+2 b$ and $\mathcal{P}_{\text {rel }}$ ensures that $\vec{\mu}_{p}^{r} \geq n-b-f$ in all rounds, line 8 is never executed and therefore can be suppressed. Furthermore, because $\mathcal{P}_{\text {rel }}$ is ensured in all rounds, we have the following property. Once a value $v$ is locked, Algorithm 3 always return $v$. By Algorithm 1, $\mathcal{P}_{r e l}$ and Selector $(p, \phi)$ always return $\Pi, v$ is always validated. This means that messages from honest processes that are in possibleVotes $_{p}$ can only be $\langle v, \phi-1,-,-\rangle$. In other words, line 1 of Algorithm 9 is equivalent to lines 1-3 of Algorithm 3.

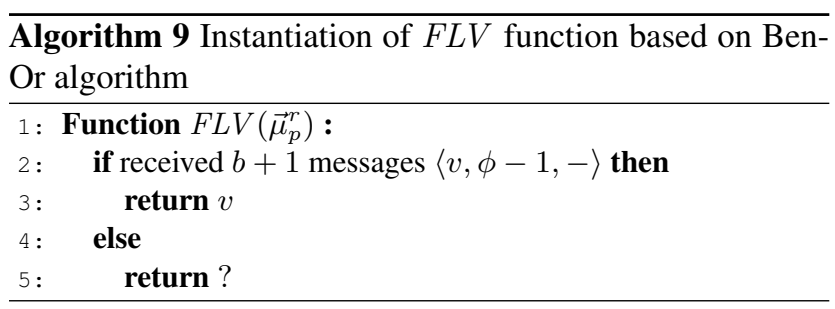

Note that in the original algorithm, a variable $t s_{p}$ is not explicitly used. It is used implicitly through the use of (1) sign ? which in instantiated algorithm corresponds to the case where $t s_{p} \neq \phi$ and (2) the sign $D$ which corresponds to the case where $t s_{p}=\phi$.

\section{Conclusion}

The paper has presented a generic consensus algorithm parameterized with $T_{D}, F L A G$, Selector and FLV. Instantiation of these parameters led us to distinguish three classes of consensus algorithms (into which known consensus algorithms fit), and to identify the new MQB algorithm. As future work, we plan to develop a framework around our generic algorithm.

Acknowledgements: We would like to thank Fatemeh Borran, Martin Hutle, Segio Mena and Nuno Santos for their comments on an earlier version of the paper.

\section{References}

[1] M. Ben-Or. Another advantage of free choice: Completely asynchronous agreement protocols. In PODC, 1983.

[2] F. Borran and A. Schiper. A Leader-free Byzantine Consensus Algorithm. To appear in ICDCN, 2010.

[3] M. Castro. Practical Byzantine fault-tolerance. $\mathrm{PhD}$ thesis. Technical report, MIT, 2000.

[4] M. Castro and B. Liskov. Practical byzantine fault tolerance and proactive recovery. ACMTCS, 2002.

[5] T. D. Chandra and S. Toueg. Unreliable failure detectors for reliable distributed systems. JACM, 1996.

[6] B. Charron-Bost and A. Schiper. The Heard-Of model: computing in distributed systems with benign failures. Distributed Computing, 22(1):49-71, 2009.

[7] C. Dwork, N. Lynch, and L. Stockmeyer. Consensus in the presence of partial synchrony. JACM, 1988.

[8] M. J. Fischer, N. A. Lynch, and M. S. Paterson. Impossibility of distributed consensus with one faulty process. JACM, 1985.

[9] R. Guerraoui and M. Raynal. The Information Structure of Indulgent Consensus. IEEE Trans. on Computers, 2004.

[10] R. Guerraoui and M. Raynal. The Alpha of Indulgent Consensus. The Computer Journal, 2006.

[11] L. Lamport. The part-time parliament. ACM Transactions on Computer Systems, 16(2):133-169, May 1998.

[12] L. Lamport, R. Shostak, and M. Pease. The Byzantine generals problem. ACM Trans. Program. Lang. Syst., 1982.

[13] B. Lampson. The abcd's of paxos. In PODC, 2001.

[14] H. C. Li, A. Clement, A. S. Aiyer, and L. Alvisi. The paxos register. In SRDS, 2007.

[15] D. Malkhi and M. K. Reiter. Byzantine quorum systems. Distributed Computing, 1998.

[16] J.-P. Martin and L. Alvisi. Fast Byzantine consensus. TDSC, 2006.

[17] Z. Milosevic, M. Hutle, and A. Schiper. Unifying Byzantine consensus algorithms with Weak Interactive Consistency. To appear in OPODIS 2009.

[18] A. Mostéfaoui, S. Rajsbaum, and M. Raynal. A versatile and modular consensus protocol. In DSN, 2002.

[19] O. Rütti, Z. Milosevic, and A. Schiper. Generic construction of consensus algorithm for benign and Byzantine faults. Technical Report LSR-REPORT-2009-005, EPFL-IC, 2009.

[20] Y. J. Song, R. van Renesse, F. B. Schneider, and D. Dolev. The building blocks of consensus. In ICDCN, 2008. 